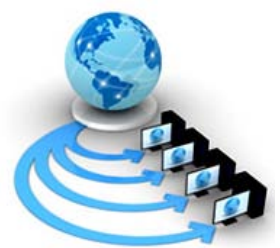

International Journal of Advanced Research in Computer Science

RESEARCH PAPER

\title{
NEURAL NETWORK BASED DEEP LEARNING AND ENSEMBLE TECHNIQUES FOR DATA CLASSIFICATION
}

\author{
K. G. Nandha Kumar \\ Doctoral Research Scholar, \\ PG \& Research Department of Computer Science, \\ Government Arts College, \\ Udumalpet, India.
}

\author{
Dr. T. Christopher \\ Assistant Professor, \\ PG \& Research Department of Computer Science, \\ Government Arts College, \\ Coimbatore, India.
}

\begin{abstract}
In this paper, four neural network architectures are proposed for data classification. The four neural networks are constructed based on deep learning and ensemble architectures. Supervised and unsupervised learning paradigms are adopted. Stack of Supervised Neural Network (SSNN), Stack of Unsupervised Neural Network (SUNN), Ensemble of Supervised Neural Network (ESNN), and Ensemble of Unsupervised Neural Network (ESNN) are the proposed neural network classifiers. Australian credit approval data set from the University of California, Irvin is used to evaluate the classifiers. Supervised neural networks have produced more accurate classification results than unsupervised networks. Stack architectures are comparatively better than ensemble architectures. It is found from this research that the combination of supervised learning method and stack architecture leads to better performance.
\end{abstract}

Keywords: Data Classification, Deep Learning, Ensembles, Neural Networks, Supervised and Unsupervised Learning.

\section{INTRODUCTION}

In the recent decades soft computing techniques have grabbed more attention of the researchers. Artificial neural networks, fuzzy logic and genetic algorithms are notable soft computing techniques and they are applied to solve various kinds of problem irrespective of domains. They are biologically inspired machine learning techniques. Supervised learning and unsupervised learning are the two significant paradigms of this soft computing approach. Deep learning neural networks and ensemble of neural networks are the advanced versions of regular methods. Deep learning can be achieved through the stack architecture and a high amount of training is required [1]. Deep neural networks are successfully applied to classification problems. Hierarchical fuzzy deep neural networks are applied for image categorization, financial data prediction and MRI segmentation of brain [2]. To improve the efficiency of deep neural networks, weight matrix parameterizing and low rank factorization are applied [3]. Diagnosis and classification of faults in semiconductor manufacturing process can be carried out by neural networks [4]. Deep convolutional features of neural network classifiers are used for classifying remote sensing images [5]. The effectiveness of deep learning in classification process is proved on remote sensing image data of lands and crops [6]. Extreme learning machines are built by stack architecture for solving various type of classification problems [7]. The complexity of deep learning neural network classifiers are discussed in the literature [8].

Application of the neural network based ensemble methods for classification problems are found better in terms of decreasing the error rate [9]. Ensembles of neural networks are applied for classification of seismic signals [10] and medical image classification [11]. Linear combination of multiple neural networks has improved the performance of classifier [12]. A combination of multiple neural networks for online pattern classification is attempted [13]. Hybrid methods such as Neuro-Fuzzy ensembles are producing better results in classification [14] [15]. Multilayer perceptron based ensembles can be used as classifiers [16] [17]. Clustering based classification is a novel approach to solve classification problems in some select domain [18]. When analyzing the literature, ensemble methods seemed better classifiers and they are applied on data related medical diagnosis also [19].

\section{PROPOSED METHODS AND EXPERIMENTS}

Australian credit approval data set is a bench mark data set provided by the UCI repository. It contains 690 records, 14 attributes and two classes. It is used here to evaluate the performance of proposed neural network architectures. The overall structure of the proposed approach is represented in Figure.1. Stack of Supervised Neural Network (SSNN), Stack of Unsupervised Neural Network (SUNN), Ensemble of Supervised Neural Network (ESNN), and Ensemble of Unsupervised Neural Network (ESNN) are the proposed neural network classifiers.

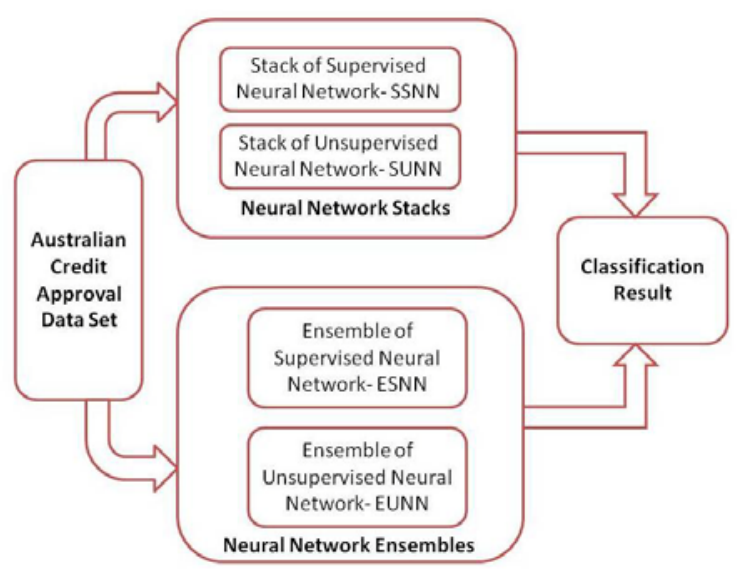

Figure.1. Functional Diagram of Proposed Approach 
The proposed methods are implemented in Python language with the specifications mentioned below.

\section{A. Stack of Supervised Neural Network (SSNN)}

Network Type : Feed forward multilayer network Learning Method : Supervised deep learning

Number of Layers: Six layers \& completely interconnected

No. of Neurons in the input Layer : Eight neurons

No. of Neurons in the hidden Layers: Eight neurons

No. of Neurons in the output Layer : One neuron

Description of layer wise task : Input weights vectors are fed into the first layer and in the second layer, the Euclidian distance among the weights is calculated to get better grouping. Perceptron learning rule is applied repeatedly in the third, the fourth and the fifth layers in order to enrich the training of the classifier. The final classification result is derived through the output layer.

\section{B. Stack of Unsupervised Neural Network (SUNN)}

Network Type : Feed forward multilayer network Learning Method : Unsupervised deep learning

Number of Layers: Six layers \& completely interconnected

No. of Neurons in the input Layer : Eight neurons

No. of Neurons in the hidden Layers: Eight neurons

No. of Neurons in the output Layer : One neuron

Description of layer wise task : Input weights vectors are fed into the first layer and in the second layer, the Euclidian distance among the weights is calculated to get better grouping. Maxnet activation function is applied repeatedly in the third, the fourth and the fifth layers in order to produce the accurate classification. The final classification result is derived through the output layer.

\section{Ensemble of Supervised Neural Network (ESNN)}

Network Type : Feed forward multilayer network Learning Method : Supervised ensemble learning

No. of Classifiers in the Ensemble : Three

Number of Layers: Four layers \& completely interconnected

No. of Neurons in the input Layer : Eight neurons

No. of Neurons in the hidden Layers: Eight neurons

No. of Neurons in the output Layer : One neuron

Description of layer wise task : Input weights vectors are fed into the first layer and in the second layer, the Euclidian distance among the weights is calculated to get better grouping. Perceptron learning rule is applied in the third layer. The final classification result is derived through the output layer. The consolidate result is produced by the ensemble at the end.

\section{Ensemble of Unsupervised Neural Network (EUNN)}

Network Type : Feed forward multilayer network Learning Method : Unsupervised ensemble learning

No. of Classifiers in the Ensemble : Three

Number of Layers: Four layers \& completely interconnected

No. of Neurons in the input Layer : Eight neurons

No. of Neurons in the hidden Layers: Eight neurons

No. of Neurons in the output Layer : One neuron

Description of layer wise task : Input weights vectors are fed into the first layer and in the second layer, the Euclidian distance among the weights is calculated to get better grouping. Maxnet activation function is applied in the third layer. The final classification result is derived through the output layer. The consolidate result is produced by the ensemble at the end.

\section{EXPERIMENTAL RESULTS}

The classification accuracy and mean squared error (MSE) rate are calculated as follows.

$$
\text { Accuracy }=(\mathrm{TP}+\mathrm{TN}) /(\mathrm{TP}+\mathrm{FP}+\mathrm{TN}+\mathrm{FN})
$$

Where, $\mathrm{TP}=$ True Positive Classification, $\mathrm{TN}=$ True Negative Classification, FP = False Positive Classification, and FN = False Negative Classification.

$$
\mathrm{MSE}=\frac{1}{\mathrm{n}} \sum_{\mathrm{i}=1}^{\mathrm{n}}\left(\widehat{\mathrm{X}_{\mathrm{i}}}-\mathrm{X}_{\mathrm{i}}\right)
$$

Where $\widehat{\boldsymbol{x}}_{\boldsymbol{t}}$ is a vector of classifications, and $\boldsymbol{x}_{\boldsymbol{i}}$ is the vector of observed values corresponding to the inputs to the function which generated the classifications.

The Figure.2. represents the performance accuracy of all the four neural network classifiers. The Figure.3. represents the mean squared error rate of all the four neural network classifiers.

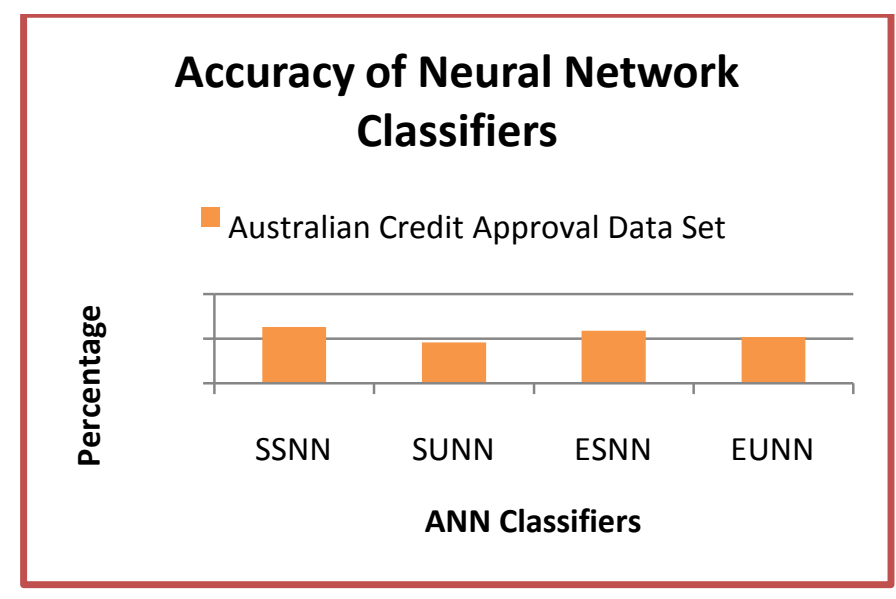

Figure.2. Accuracy of proposed classifiers.

\section{Mean Squared Error of Classifiers}

- Australian Credit Approval Data Set

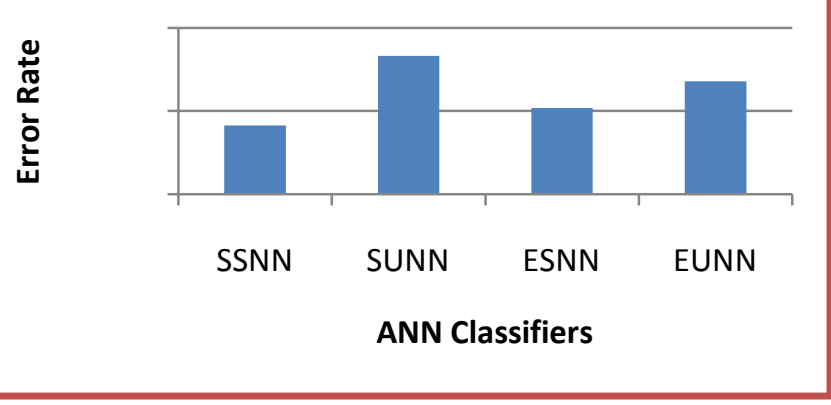

Figure.3. Error rate of proposed classifiers. 


\section{CONCLUSION AND FUTURE SCOPE}

The obtained classification accuracy of the proposed SSNN, SUNN, ESNN and EUNN are 86.3\%, 84.6\%, 85.9\% and $85.2 \%$ respectively. The error rate of SSNN, SUNN, ESNN and EUNN are $0.1365,0.1533,0.1408$, and 0.1472 respectively. Results indicate that among the proposed techniques, supervised methods are more efficient in classification when compared with unsupervised neural network. In future researches, hybrid soft computing techniques such as Neuro-fuzzy, Neuro-genetic, fuzzy-genetic may be applied to improve the performance of classifiers.

\section{REFERENCES}

[1] Y. Furusho, T. Kubo, and K. Ikeda, "Roles of pre-training in deep neural networks from information theoretical perspective,” Neurocomputing, vol. 248, pp. 76-79, 2017.

[2] Y. Deng, Z. Ren, Y. Kong, F. Bao, and Q. Dai, “A Hierarchical Fused Fuzzy Deep Neural Network for Data Classification,” IEEE Trans. Neural Networks Learn. Syst., vol. 6706, no. c, pp. 1-8, 2016.

[3] B. Chandra and R. K. Sharma, "Fast learning in Deep Neural Networks," Neurocomputing, vol. 171, pp. 12051215, 2016.

[4] K. B. Lee, S. Cheon, and C. O. Kim, “A Convolutional Neural Network for Fault Classification and Diagnosis in Semiconductor Manufacturing Processes," IEEE Trans. Semicond. Manuf., vol. 6507, no. c, pp. 1-1, 2017.

[5] Q. Weng, Z. Mao, J. Lin, and W. Guo, "Land-Use Classification via Extreme Learning Classifier Based on Deep Convolutional Features,” pp. 1-5, 2017.

[6] N. Kussul, M. Lavreniuk, S. Skakun, and A. Shelestov, "Deep Learning Classification of Land Cover and,” pp. 15, 2017.

[7] H. Zhou, G.-B. Huang, Z. Lin, H. Wang, and Y. C. Soh, "Stacked Extreme Learning Machines.," IEEE Trans. Cybern., vol. PP, no. 99, p. 1, 2014.
[8] M. Bianchini and F. Scarselli, "On the complexity of shallow and deep neural network classifiers,” Esann 2014, vol. 25, no. April, pp. 23-371-376, 2014.

[9] L. K. Hansen and P. Salamon, "Neural Network Ensembles,” IEEE Trans. Pattern Anal. Mach. Intell., vol. 12, no. 10, pp. 993-1001, 1990.

[10] Y. Shimshoni and N. Intrator, "Classification of seismic signals by integrating ensembles of neural networks," IEEE Trans. Signal Process., vol. 46, no. 5, pp. 1194-1201, 1998.

[11] A. Kumar, J. Kim, D. Lyndon, M. Fulham, and D. Feng, "An Ensemble of Fine-Tuned Convolutional Neural Networks for Medical Image Classification,” IEEE J. Biomed. Heal. Informatics, vol. 21, no. 1, pp. 1-1, 2016.

[12] N. Ueda, "Optimal linear combination of neural networks for improving classification performance,” Pattern Anal. Mach. Intell. IEEE Trans., vol. 22, no. 2, pp. 207-215, 2000.

[13] C. P. Lim and R. F. Harrison, "Network SystemsAn Experimental Study,” vol. 33, no. 2, pp. 235-247, 2003.

[14] C. K. Loo and M. V. C. Rao, "Accurate and reliable diagnosis and classification using probabilistic ensemble simplified fuzzy ARTMAP," IEEE Trans. Knowl. Data Eng., vol. 17, no. 11, pp. 1589-1593, 2005.

[15] H. H. Dam, H. A. Abbass, S. Member, C. Lokan, and X. Y. Fellow, "Neural-Based Learning Classifier Systems," Knowl. Creat. Diffus. Util., vol. X, no. Xx, pp. 1-14, 2007.

[16] T. Windeatt, "Ensemble MLP classifier design," Stud. Comput. Intell., vol. 137, no. 5, pp. 133-147, 2008.

[17] T. Windeatt, R. Duangsoithong, and R. Smith, "Embedded feature ranking for ensemble MLP classifiers,” IEEE Trans. Neural Networks, vol. 22, no. 6, pp. 988-994, 2011.

[18] A. Rahman and B. Verma, "Novel layered clustering-based approach for generating ensemble of classifiers," IEEE Trans. Neural Networks, vol. 22, no. 5, pp. 781-792, 2011.

[19] S. Huda, J. Yearwood, H. F. Jelinek, M. M. Hassan, and G. Fortino, "A Hybrid Feature Selection With Ensemble Classification for Imbalanced Healthcare Data: A Case Study for Brain Tumor Diagnosis,” vol. 4, 2017. 